\title{
MELNIKOV ANALYSIS OF A SHIP'S STABILITY WITH WATER-ON-DECK
}

\section{Ying-Guang Wang}

Department of Naval Architecture and Ocean Engineering, Shanghai Jiao Tong University, 800 Dong Chuan Road, Shanghai, P.R.C

Follow this and additional works at: https://jmstt.ntou.edu.tw/journal

Part of the Transportation Engineering Commons

\section{Recommended Citation}

Wang, Ying-Guang (2010) "MELNIKOV ANALYSIS OF A SHIP'S STABILITY WITH WATER-ON-DECK," Journal of Marine Science and Technology: Vol. 18: Iss. 1, Article 13.

DOI: $10.51400 / 2709-6998.1871$

Available at: https://jmstt.ntou.edu.tw/journal/vol18/iss1/13

This Research Article is brought to you for free and open access by Journal of Marine Science and Technology. It has been accepted for inclusion in Journal of Marine Science and Technology by an authorized editor of Journal of Marine Science and Technology. 


\section{MELNIKOV ANALYSIS OF A SHIP'S STABILITY WITH WATER-ON-DECK}

Acknowledgements

Thanks are due to Ms. Bian Songping for having provided valuable assistance with the editorial work for this article. Her carefulness and expertise are very much appreciated 


\title{
MELNIKOV ANALYSIS OF A SHIP'S STABILITY WITH WATER-ON-DECK
}

\author{
Ying-Guang Wang*
}

Key words: ship stability criterion, Melnikov function, phase plane diagrams, Poincare maps.

\begin{abstract}
The nonlinear ship rolling motion with water-on-deck in regular beam waves is studied by utilizing a global geometric method. A ship stability criterion based upon the Melnikov function is obtained to provide an upper bound on the domain of the potential chaotic rolling motion. Phase plane diagrams and Poincare maps are used to validate the ship stability criterion obtained in this article.
\end{abstract}

\section{INTRODUCTION}

In this article we investigate the important naval architectural problem of vessel rolling with water trapped on deck. The presence of water on deck can significantly change the behavior of a ship. One way to investigate the effects of water-on-deck is by conducting wave tank model experiments. Adee and Pantazopoulos [1] have carried out ship model tests to predict the water-on-deck effects. Their experiments suggested the importance of the so-called pseudo-static heel angle (loll angle) when water is trapped on deck. However, physical experiments involve unknowns due to scaling and are extremely costly. Thus, they are limited in the number of parameters that can be systematically studied.

Another method to study the water-on-deck effects is numerical simulation [2], i.e. to perform numerical integration on the vessel's differential equation of motion. Simulation has the great advantage of being able to conveniently deal with any type of nonlinearities. However, the numerical simulation technique is very time consuming [13] so that fully solving a given ship stability problem, although theoretically possible, may be impractical. In addition, simulations rely heavily on accurate prediction of initial conditions of the ship motion.

Geometric methods have been recognized in the engineering community for the analysis of nonlinear dynamics exhibiting chaotic behavior. Instead of directly solving the nonlinear

Paper submitted 02/20/09; revised 03/12/09; accepted 03/13/09. Author for correspondence: Ying-Guang Wang (e-mail: wyg110@sjtu.edu.cn).

*Department of Naval Architecture and Ocean Engineering, Shanghai Jiao Tong University, 800 Dong Chuan Road, Shanghai, P.R.C. differential equation of motion, the geometric method emphasizes the qualitative behavior of the system, or more precisely, the changes in qualitatively different behaviors. One of the significant analytic results of the geometric method is the Melnikov function $[7,16]$ which can predict the occurrence of chaos in a certain class of systems. Falzarano et al. [5] first utilized global geometric analysis techniques to study transient rolling motions of a small ship with water-on-deck which is subjected to a periodic wave excitation. A linear-plus-quadratic type damping $\operatorname{term}\left(B^{\prime}{ }_{44} \dot{\phi}+B_{44 q} \dot{\phi}|\dot{\phi}|\right)$ was used in the equation of motion in their study. Their analysis is based on determining a Melnikov criterion which can predict the qualitative nature of the invariant manifolds which represent the boundary between safe and unsafe initial conditions, and how these depend on system parameters for the small ship model. Of particular interest is the transition which this boundary makes from regular to fractal, implying a loss in predictability of the ship's eventual state. Wang and Tan [14] recently extended the global geometric analysis techniques [5] in analyzing the nonlinear rolling motion of a biased ship (possibly due to water-on-deck) in random waves. They followed Falzarano et al. [5] to use a linear-plus-quadratic type damping term $\left(B^{\prime}{ }_{44} \dot{\phi}+B_{44 q} \dot{\phi}|\dot{\phi}|\right)$ in the equation of motion for their study. A mean-square Melnikov criterion is obtained in their study to provide an upper bound on the domain of the potential chaotic rolling motion.

In the present study, we will use a linear-plus-cubic type damping term $\left(B^{\prime}{ }_{44} \dot{\phi}+B_{3} \dot{\phi}^{3}\right)$ in the equation of motion for studying the ship rolling motion with water-on-deck (Liu and Tang [11]). We prefer to use the linear-plus-cubic type damping term because the rolling equation with this term retains the essential dynamics of the physical system as has been indicated by model experiments (Spyrou, et al. [12]; Francescutto and Contento [6]). A ship stability criterion based upon the Melnikov function will be obtained in this article to provide an upper bound on the domain of the potential chaotic rolling motion (erratic rocking). Moreover, the present work will make a first effort to systematically change the system parameters in the rolling equation and use phase plane diagrams and Poincare maps to validate the Melnikov criterion obtained in this article. 


\section{PHYSICAL MODELING}

Because of port-starboard symmetry of a ship, the first-order couplings from surge, heave and pitch to roll are all zero. However, the couplings from sway and yaw to roll are not. In this study, the yaw coupling with roll and sway is assumed to be small, and therefore only the coupling between sway and roll is considered. We start with the linear two degree of freedom equations of motion involving roll and sway [9]:

$$
\begin{gathered}
{\left[\begin{array}{cc}
M+A_{22} & A_{24}-M z_{c} \\
A_{42}-M z_{c} & I_{4}+A_{44}
\end{array}\right]\left[\begin{array}{l}
\ddot{\eta} \\
\ddot{\phi}
\end{array}\right]+\left[\begin{array}{cc}
B_{22} & B_{24} \\
B_{42} & B_{44}
\end{array}\right]\left[\begin{array}{l}
\dot{\eta} \\
\dot{\phi}
\end{array}\right]} \\
+\left[\begin{array}{cc}
0 & 0 \\
0 & C_{44}
\end{array}\right]\left[\begin{array}{l}
\eta \\
\phi
\end{array}\right]=\left[\begin{array}{l}
F_{2}(t) \\
F_{4}(t)
\end{array}\right]
\end{gathered}
$$

where $\eta$ represents the sway displacement, $\phi$ represents the roll angle. $M$ is the mass of the ship. $I_{4}$ is the moment of inertia (in air) of the ship about the roll axis. The $A$ 's and $B$ 's are added mass and damping coefficients which can be calculated from any linear hydrodynamic program, e.g. TRIBON. $z_{c}$ is the vertical center of gravity of the ship from the base line. $C_{44}$ is the roll-restoring moment coefficient. $F_{2}(t)$ and $F_{4}(t)$ are the external wave exciting force and moment, respectively. Further, the symbol $(\bullet)$ denotes differentiation with respect to time $t$.

In general, the roll motion and sway cannot be decoupled because of damping. For special cases, i.e., undamped or proportionally damped systems, it can be shown that the ship rolls about a roll center like a pendulum, and the roll motion can be decoupled from sway. If a pseudo roll center is assumed to exist when general damping is present, we then get the following single degree of freedom roll equation [13]:

$$
\left[I^{\prime}{ }_{44}+A^{\prime}{ }_{44}\right] \ddot{\phi}+B^{\prime}{ }_{44} \dot{\phi}+C^{\prime}{ }_{44} \phi=F(t)
$$

where

$$
\begin{gathered}
I^{\prime}{ }_{44}=I_{4}+A_{42} R_{c}, \quad A_{44}^{\prime}=A_{44}-M z_{c} R_{c}, \\
B_{44}^{\prime}=B_{44}+B_{24} R_{C}, \quad R_{C}=-\frac{A_{42}-M z_{c}}{M+A_{22}}, \\
F(t)=F_{4}(t)+F_{2}(t) R_{c}, \quad C^{\prime}{ }_{44} \phi=\Delta G Z_{m}(\phi)
\end{gathered}
$$

In the above equation, $\Delta$ is the displacement of the ship, $G Z_{m}(\phi)$ is a modified polynomial approximation to the nonlinear roll-restoring arm. Caglayan [3] suggests that the dominant dynamics of the water-on-deck problem can be approximated by a fixed weight to achieve the same pseudostatic heel angle (loll angle) of the ship. Therefore, the $G Z_{m}(\phi)$ curve can be obtained by modifying the original roll-restoring arm curve $G Z(\phi)$ after considering the effects of weight and moment of water-on-deck. Following the work of Falzarano [4], we use a third order polynomial to approximate the lolled righting arm curve (the part ahead of the loll angle):

$$
G Z_{m}(\phi)=-C_{1} \phi+C_{3} \phi^{3}
$$

in which the constants $C_{1}$ and $C_{3}$ can be calculated by using a ship hydrostatic stability program (e.g. TRIBON) and a suitable interpolation procedure.

We know that the rolling motion around the resonance condition is strongly affected by the viscous damping, and the viscous roll damping is a nonlinear function of the rolling velocity. Therefore, a cubic typed viscous damping term should be added in (2):

$$
\left[I^{\prime}{ }_{44}+A^{\prime}{ }_{44}\right] \ddot{\phi}+B^{\prime}{ }_{44} \dot{\phi}+B_{3} \dot{\phi}^{3}+\Delta G Z_{m}(\phi)=F(t)
$$

Despite its simplicity, the above equation retains the essential dynamics of the physical system as has been indicated by model experiments (Spyrou et al. [12]; Francescutto and Contento [6]). In (4), $B_{3}$ can be calculated by utilizing some empirical formulae. For harmonic excitation in regular seas, $F(t)$ in (4) is calculated as:

$$
F(t)=A F_{\text {roll }}(\omega) \cos (\omega t)
$$

where $A$ is the wave amplitude, and $F_{\text {roll }}(\omega)$ is the moment amplitude per unit wave amplitude at frequency $\omega$.

To study the dynamic stability of a ship, the traditional way is to perform numerical integration on the nonlinear differential equation of motion (4) via a suitable numerical procedure, e.g. the fourth order Runge Kutta method. However, an inexperienced numerical analyst could integrate (4) for a long time and never discover the most important or critical behavior.

The Melnikov method can offer a new way to the naval architects for analyzing the dynamic stability of a ship, and the method is based on the modern nonlinear dynamics theory. An important result obtained in the process of analyzing a ship's dynamic stability via the Melnikov method is the Melnikov criterion. In some cases, this criterion can directly link the ship design parameters to the wave characteristic parameters by a simple analytical formula. This will greatly enhance the efficiency for rationally analyzing the dynamic stability of a ship. Even if in some cases we can not obtain the analytical expression of the Melnikov function, we can still numerically integrate the Melnikov integral in a straightforward manner. In the next section we will derive a Melnikov criterion for studying the nonlinear ship rolling with water-on-deck.

\section{MELNIKOV ANALYSIS}

The Melnikov method is used to calculate the distance 
between the stable and unstable manifolds and to detect transverse homoclinic intersections. The Melnikov method determines the distance between these two manifolds by beginning with a dynamical system where the homoclinic solutions are known, and perturbing this system slightly to determine what happens to these manifolds as a result of the perturbation. This procedure results in a formula or criterion for the distance between the stable and unstable manifolds in terms of the system parameters and the phase.

In order to conveniently apply the Melnikov method for analyzing the ship rolling with water-on-deck, we first change (4) into a non-dimensional form:

$$
\ddot{x}(\tau)+\varepsilon \delta \dot{x}(\tau)+\varepsilon \delta_{3} \dot{x}^{3}(\tau)-x(\tau)+k x^{3}(\tau)=\varepsilon \gamma \cos (\Omega \tau)(6)
$$

where:

$$
\begin{gathered}
x=\phi \quad \tau=\omega_{n} t \\
\omega_{n}=\sqrt{\frac{C_{1} \Delta}{I^{\prime}{ }_{44}+A^{\prime}{ }_{44}}} \quad \Omega=\frac{\omega}{\omega_{n}} \\
\varepsilon \delta=\frac{B_{44}^{\prime} \omega_{n}}{C_{1} \Delta} \quad \varepsilon \delta_{3}=\frac{B_{3} \sqrt{C_{1} \Delta}}{\left(I^{\prime}{ }_{44}+A^{\prime}{ }_{44}\right)^{3 / 2}} \\
k=\frac{C_{3}}{C_{1}} \quad \varepsilon \gamma=\frac{A F_{\text {roll }}(\omega)}{C_{1} \Delta}
\end{gathered}
$$

The above derivation procedure basically follows that of Wang and Tan [15] on analyzing a barge's dynamic stability by using the Melnikov method. In (6), time is scaled by the linear natural frequency, $\omega_{n}$. The differentiation in (6) is with respect to time $\tau$. The terms indicated by $\varepsilon$ are taken to be small and will be treated as perturbations in the following analysis. This treatment has already been justified by the numerical values of the coefficients for some real ships used in the studies of several authors (Wang and Tan [15]; Hsieh et al. [8]; Jiang et al. [10]).

Let $x=\mathrm{Q}, \dot{x}=\mathrm{P}$, Eq. (6) can be rewritten into the form of a perturbed Hamiltonian system:

$$
\left\{\begin{array}{l}
\dot{Q}=\frac{\partial H}{\partial P}=P \\
\dot{P}=-\frac{\partial H}{\partial Q}+\varepsilon\left[-\delta \frac{\partial H}{\partial P}-\delta_{3}\left(\frac{\partial H}{\partial P}\right)^{3}+\gamma \cos (\Omega \tau)\right]
\end{array}\right.
$$

The Hamiltonian corresponding to the unperturbed system $\ddot{x}(\tau)-x(\tau)+k x^{3}(\tau)=0$ is:

$$
H(Q, P)=\frac{1}{2} \dot{x}^{2}-\frac{1}{2} x^{2}+\frac{k}{4} x^{4}
$$

The well known expressions of the homoclinic orbits of the unperturbed Hamiltonian system $\ddot{x}(\tau)-x(\tau)+k x^{3}(\tau)=0$ are:

$$
Q_{0}(\tau)=\sqrt{\frac{2}{k}} \operatorname{sech}(\tau), P_{0}(\tau)=\sqrt{\frac{2}{k}}(-\operatorname{sech}(\tau) \tanh (\tau))
$$

An unperturbed ship system with no water-on-deck has a stable upright equilibrium, $(x, \dot{x})=(0,0)$. Changing from the no water-on-deck situation to the static effect of substantial water-on-deck, the slope of the ship's righting arm curve at the origin, the so-called metacentric height GM, is reduced through zero to a minus value. When this occurs, the stable upright equilibrium, $(x, \dot{x})=(0,0)$ bifurcates into an unstable upright equilibrium and two stable equilibria at the positive and negative loll angles, $\pm x_{l}$. This bifurcation is the classical pitchfork bifurcation. The bifurcated system's phase portrait near the loll angles, $\pm x_{l}$ will have two homoclinic orbits (as described by (9)), each connecting the unstable saddle at the origin to itself. The two homoclinic orbits consist of the stable and unstable manifolds. For an unperturbed (undamped and unforced) ship system the stable and unstable manifolds coincide.

The Melnikov function for the perturbed system (7) can be calculated as follows (Wang and Tan [15]):

$$
\begin{aligned}
& M\left(\tau_{0}\right) \\
& =\int_{-\infty}^{\infty} \frac{\partial H}{\partial P}\left[-\delta \frac{\partial H}{\partial P}-\delta_{3}\left(\frac{\partial H}{\partial P}\right)^{3}+\gamma \cos \left(\Omega\left(\tau+\tau_{0}\right)\right)\right] d \tau \\
& =-\delta \int_{-\infty}^{\infty}\left(\frac{\partial H}{\partial P}\right)^{2} d \tau-\delta_{3} \int_{-\infty}^{\infty}\left(\frac{\partial H}{\partial P}\right)^{4} d \tau \\
& \quad+\int_{-\infty}^{\infty} \frac{\partial H}{\partial P} \gamma \cos \left(\Omega\left(\tau+\tau_{0}\right)\right) d \tau \\
& =\bar{M}\left(\tau_{0}\right)-\bar{M}
\end{aligned}
$$

where:

$$
\begin{gathered}
\bar{M}=\delta \int_{-\infty}^{\infty}\left(\frac{\partial H}{\partial P}\right)^{2} d \tau+\delta_{3} \int_{-\infty}^{\infty}\left(\frac{\partial H}{\partial P}\right)^{4} d \tau \\
\bar{M}\left(\tau_{0}\right)=\int_{-\infty}^{\infty} \frac{\partial H}{\partial P} \gamma \cos \left(\Omega\left(\tau+\tau_{0}\right)\right) d \tau
\end{gathered}
$$

are, respectively, the mean and oscillatory part of the Melnikov function. Substituting (9) into the above equations yields:

$$
\bar{M}=\frac{4}{3 k} \delta+\frac{16}{35 k^{2}} \delta_{3}
$$

$$
\bar{M}\left(\tau_{0}\right)=\sqrt{\frac{2}{k}}(\gamma \pi \Omega) \operatorname{Sin}\left(\Omega \tau_{0}\right) \operatorname{Sech}\left(\frac{\pi \Omega}{2}\right)
$$


Then, the complete expression of the Melnikov function is:

$$
M\left(\tau_{0}\right)=\sqrt{\frac{2}{k}}(\gamma \pi \Omega) \operatorname{Sin}\left(\Omega \tau_{0}\right) \operatorname{Sech}\left(\frac{\pi \Omega}{2}\right)-\frac{4}{3 k} \delta-\frac{16}{35 k^{2}} \delta_{3}
$$

The condition at which the Melnikov function has simple zeros is:

$$
\sqrt{\frac{2}{k}}(\gamma \pi \Omega) \operatorname{Sech}\left(\frac{\pi \Omega}{2}\right)-\frac{4}{3 k} \delta-\frac{16}{35 k^{2}} \delta_{3}=0
$$

When damping and external excitations are added to the unperturbed system, the perturbed stable and unstable manifolds will no longer coincide. The distance that separates them depends upon the position on the unperturbed manifolds, and is called the Melnikov distance. To first order, the Melnikov distance is proportional to the absolute value of the Melnikov function calculated by (15). If the Melnikov function has simple zeros, the stable and unstable manifolds intersect transversely. By a theorem attributed to Poincaré (Guckenheimer and Holmes [7]), if the stable and unstable manifolds cross each other once, they will intersect an infinite number of times, thus forming homoclinic tangles.

Here for illustration purpose only we choose the system parameters as $\Omega=1, k=1, \delta=0.15$ and $\delta_{3}=0.3$. Equation (16) leads to a critical value of the wave excitation amplitude of $\gamma=0.153$ at which homoclinic tangles are created simultaneously on both sides of the origin, due to the symmetry. Chaos will likely to occur at forcing amplitudes above the critical value. Here it should be noticed that the Melnikov boundary is a necessary but not a sufficient condition for observed chaos (i.e., it is a lower bound). For wave amplitudes above the critical, the ship dynamics resulting from the homoclinic intersection would be an erratic oscillation between the two loll angles and loss of prediction of which side the ship will lean to. Although this is not a disastrous behavior, it is very disturbing.

\section{PHASE PLANE DIAGRAMES AND POINCARE MAPS}

The ship rolling equation of motion (6) is also studied by using phase plane diagrams and Poincare maps. Keeping the system parameters $\Omega=1, k=1, \delta=0.15$ and $\delta_{3}=0.3$ unchanged, we systematically vary the value of the wave excitation amplitude $\gamma$ in (6) to study the motion responses of the vessel. We first choose a value of $\gamma=0.15$ which is smaller than the critical wave excitation amplitude predicted by the Melnikov criterion. Equation (6) is then numerically integrated in a time range of $(0,100)$ with the initial conditions randomly chosen to be $(x(0), \dot{x}(0))=(1.45,0.5)$. The obtained phase plane diagram is shown in Fig. 1(a). We see from

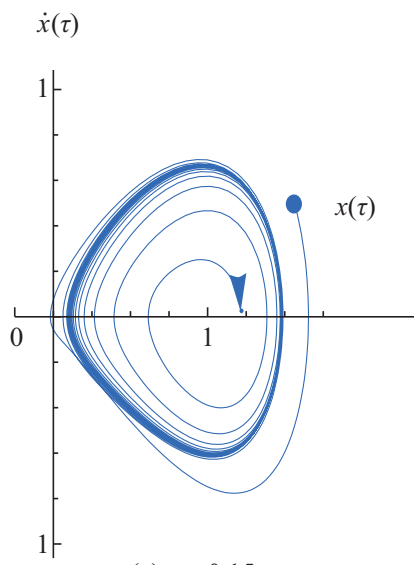

(a) $\gamma=0.15$

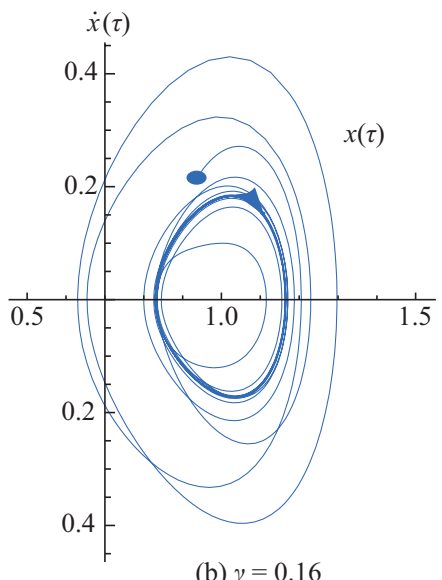

(b) $\gamma=0.16$

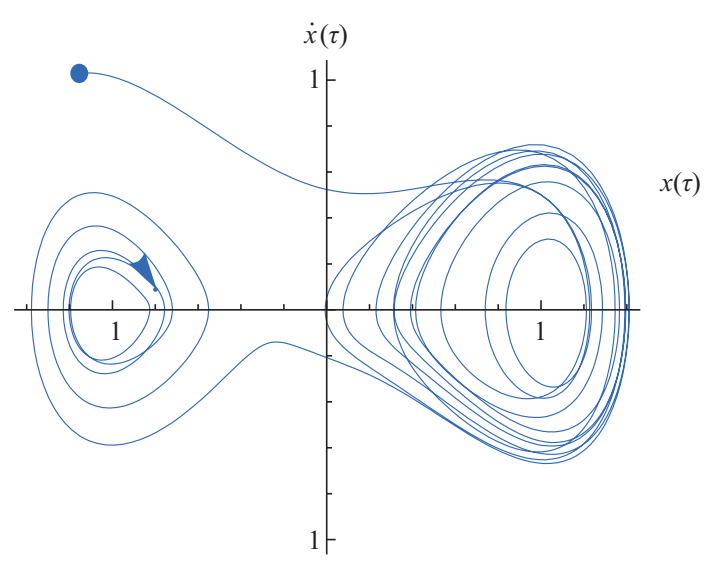

(c) $\gamma=0.2$

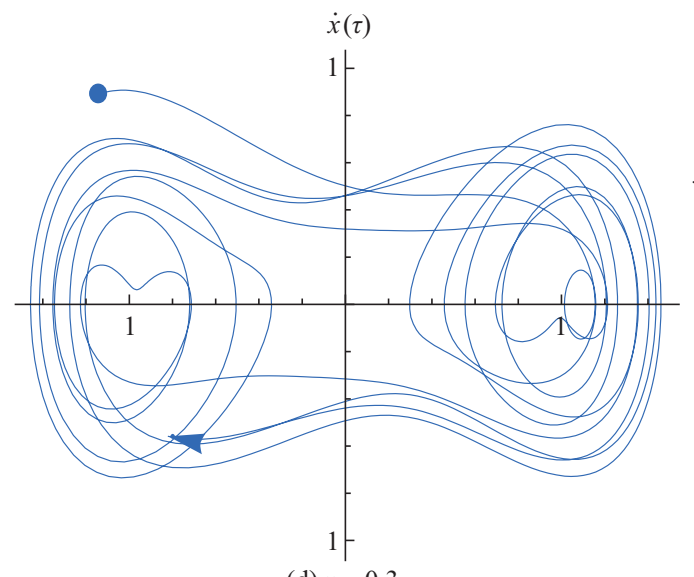

(d) $\gamma=0.3$

Fig. 1. phase plane diagrams of the ship rolling equation of motion (6) when (a) $\gamma=0.15$; (b) $\gamma=0.16$; (c) $\gamma=0.2$; (d) $\gamma=0.3$.

Fig. 1(a) that the trajectories of the ship rolling responses are quite regular. Next we increase the value of the wave excitation amplitude to $\gamma=0.16$ which is slightly bigger than the 
critical wave excitation amplitude predicted by the Melnikov criterion. Equation (6) is again numerically integrated in a time range of $(0,100)$ with the initial conditions randomly chosen to be $(x(0), \dot{x}(0))=(0.9375,0.216)$. The obtained phase plane diagram is shown in Fig. 1(b). We find that the ship rolling response trajectories start to get intersected. To systematically study the influence of the wave excitation amplitude on the ship response, we continually choose two values $\gamma=0.2$ and $\gamma=0.3$, and numerically integrated (6) in a time range of $(0,100)$. The obtained phase plane diagrams are shown in Figs. 1(c) and 1(d). We notice that the trajectories of the ship rolling responses are becoming messy and hard to interpret.

We finally utilize Poincare maps to study the ship rolling and validate the Melnikov criterion obtained in Section III. Keeping the system parameters $\Omega=1, k=1, \delta=0.15$ and $\delta_{3}=$ 0.3 unchanged, we first choose a value of $\gamma=0.15$ which is smaller than the critical wave excitation amplitude predicted by the Melnikov criterion. Equation (6) is then numerically integrated in a time range of $(1000,10000)$ with the initial conditions randomly chosen to be $(x(1000), \dot{x}(1000))=(0.5$, 0.4). The obtained Poincare map is shown in Fig. 2(a). We then use a value of $\gamma=0.16$ which is slightly larger than the critical wave excitation amplitude predicted by the Melnikov criterion. Integrating (6) in a time range of $(1000,10000)$ with the initial conditions randomly chosen to be $(x(1000)$, $\dot{x}(1000))=(0.9375,0.216)$, a Poincare map is again obtained and is shown in Fig. 2(b). It is seen from Fig. 2(b) that the system has not become chaotic at this moment. There are 6-7 points clustered together in a small region of the phase plane, indicating that the system is still quasi-periodic. However, this situation does not contradict the result predicted with our Melnikov criterion in Section 3 since the Melnikov boundary is a necessary but not a sufficient condition for observed chaos (i.e., it is a lower bound). As we continue to increase the wave excitation amplitude value to $\gamma=0.2$, a chaotic attractor looms to appear as shown in Fig. 2(c). The time integration range of $(100,10000)$ and the initial conditions $(x(100), \dot{x}(100)=$ $(-1.16,1.02)$ are used for getting the Fig. 2(c). Please notice that we have intentionally utilized a different kind of color for the Poincare map in Fig. 2(c) to make it distinguishable. Finally, when the wave excitation amplitude value is increased to be $\gamma=0.3$, a clear picture of a chaotic attractor has been obtained and is shown as Fig. 2(d). To get this chaotic attractor, the initial conditions $(x(1000), \dot{x}(1000)=(-0.77,0.39)$ and a time integration range of $(1000,10000)$ are adopted.

The above numerical integration results qualitatively confirm the conclusion drawn by using our Melnikov criterion in Section III. For wave amplitudes above the critical, the chaotic ship dynamics predicted using the Melnikov criterion would be an erratic oscillation between the two loll angles and loss of prediction of which side the ship will lean to. Therefore, it can be said that the Melnikov criterion in this article can yield meaningful results for predicting ship erratic rocking when water is trapped on deck.
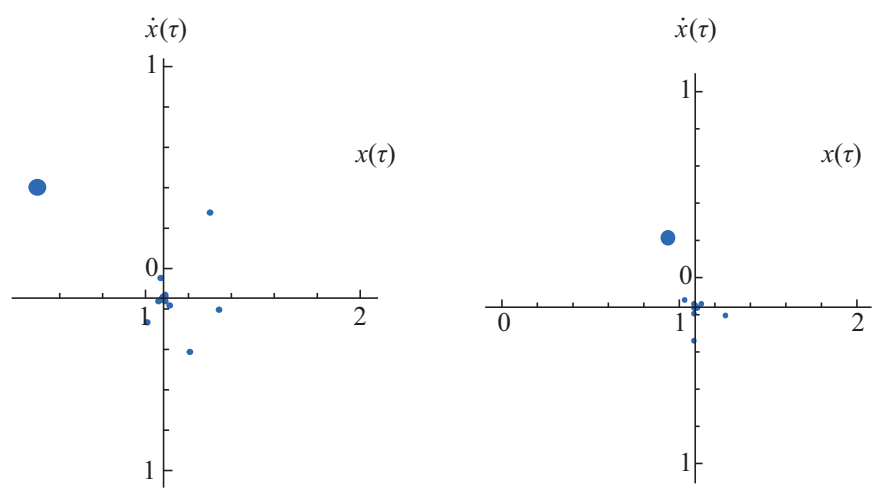

(a) $\gamma=0.15$

(b) $\gamma=0.16$

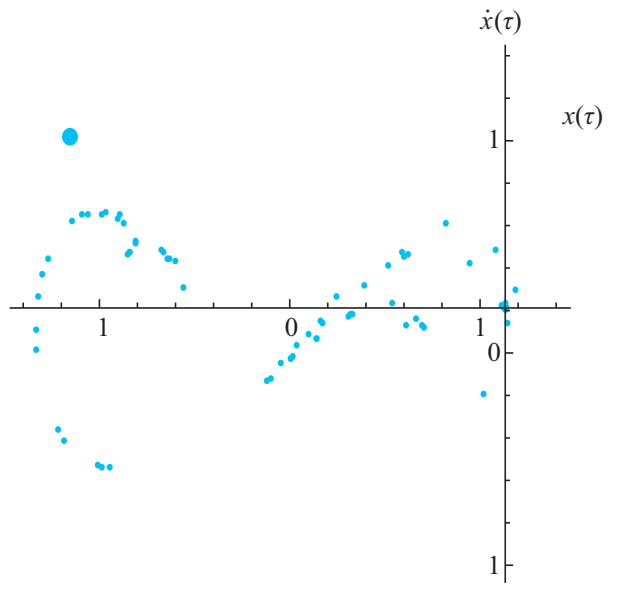

(c) $\gamma=0.2$

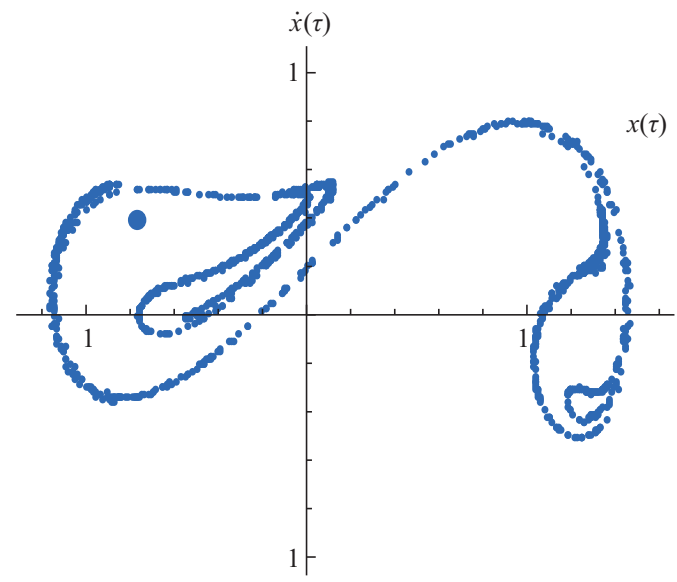

(d) $\gamma=0.3$

Fig. 2. Poincare maps of the ship rolling equation of motion (6) when (a) $\gamma=0.15$; (b) $\gamma=0.16$; (c) $\gamma=0.2$; (d) $\gamma=0.3$.

\section{CONCLUSION}

In this article, a nonlinear equation of motion with cubic typed viscous damping term has been established for studying 
the ship rolling motion with water-on-deck in regular beam waves. A ship stability criterion based upon the Melnikov function is obtained to provide an upper bound on the domain of the potential chaotic rolling motion. Physically, the chaotic ship dynamics predicted by using the Melnikov criterion will be an erratic oscillation between the two loll angles and loss of prediction of which side the ship will lean to.

To validate the Melnikov criterion obtained in this article, the value of the wave excitation amplitude in the equation of motion of the ship is systematically varied, and the equation of motion is numerically integrated to obtain the phase plane diagrams and Poincare maps. The numerical integration results qualitatively confirm the conclusion drawn by using our Melnikov criterion. It can be concluded that the Melnikov criterion in this article can yield meaningful results for predicting ship erratic rocking when water is trapped on deck.

\section{ACKNOWLEDGMENT}

Thanks are due to Ms. Bian Songping for having provided valuable assistance with the editorial work for this article. Her carefulness and expertise are very much appreciated.

\section{REFERENCES}

1. Adee, H. B. and Pantazopoulos, M. S., "Experimental study of a vessel response in waves with water trapped on deck," Proceedings of the $3 \mathrm{rd}$ International Conference on the Stability of Ships and Ocean Vehicles, Gdansk, Poland. (1986).

2. Belenky, V., Liut, D., Weems, K., and Shin, Y. S., "Nonlinear ship roll simulation with water-on-deck," Proceedings of the Stability Workshop, Glen Cove, NY. (2002).

3. Caglayan, I. H., "Water-on-deck: A theoretical and experimental study," SNAME, Texas. (1985)

4. Falzarano, J. M., Predicting Complicated Dynamics Leading to Vessel Capsizing, PhD dissertation, Department of Naval Architecture and Ma- rine Engineering, the University of Michigan. (1990).

5. Falzarano, J. M., Shaw, S. W., and Troesch, A. W., "Application of global methods for analyzing dynamical systems to ship rolling motion and capsizing," International Journal of Bifurcation and Chaos [in Applied Sciences and Engineering], Vol. 2, No. 1, pp. 101-115 (1992).

6. Francescutto, A. and Contento, G., "The modeling of the excitation of large amplitude rolling in beam waves," Proceedings, 4th International Ship Stability Workshop, St. Johns, Newfoundland, Canada, p. 6 (1998).

7. Guckenheimer, J. and Holmes, P., Nonlinear Oscillations, Dynamical Systems, and Bifurcations of Vector Fields, Applied Mathematical Sciences, Springer, v. 42 (2002).

8. Hsieh, S. R., Troesch, A. W., and Shaw, S. W., "Nonlinear probabilistic method for predicting vessel capsizing in random beam seas," Proceedings of The Royal Society of London, Series A: Mathematical and Physical Sciences, Vol. 446, pp. 195-211 (1994).

9. Jiang, C. B., Highly Nonlinear Rolling Motion Leading to Capsize, $\mathrm{PhD}$ dissertation, Department of Naval Architecture and Marine Engineering, the University of Michigan. (1995).

10. Jiang, C. B., Troesch, A. W., and Shaw, S. W., "Capsize criteria for ship models with memory-dependent hydrodynamics and random excitation," Philosophical Transactions of the Royal Society of London, A 358, pp. 1761-1791 (2000).

11. Liu, L. Q. and Tang, Y. G., "Stability of ships with water on deck in random beam waves," Journal of Vibration and Control, Vol. 13, pp. 269-280 (2007).

12. Spyrou, K. J., Cotton, B., and Gurd, B., “Analytical expressions of capsize boundary for a ship with roll bias in beam waves," Journal Ship Research, Vol. 46, No. 3, pp. 167-174 (2002).

13. Wang, Y. G., Research on Slow Drift Extreme Response and Stability of Ocean Structures in Random Seas, Ph.D dissertation, School of Naval Architecture, Ocean and Civil Engineering, Shanghai Jiao Tong University (in Chinese) (2008).

14. Wang, Y. G. and Tan, J. H., "Global geometric analysis of ship rolling and capsizing in random waves," China Ocean Engineering, Vol. 21, No. 4, pp. 577-586 (2007)

15. Wang, Y. G. and Tan, J. H., "Analyzing an ocean structure's dynamic stability by using the Melnikov method," Ocean Engineering (in press, in Chinese) (2009).

16. Wiggins, S., Introduction to Applied Non-linear Dynamical Systems and Chaos, 2nd ed., Springer (2003). 